\title{
Retinal microvascular abnormalities overlying choroidal nodules in neurofibromatosis type 1
}

\author{
Solmaz Abdolrahimzadeh ${ }^{1 *}$, Lorenzo Felli ${ }^{2}$, Domenica Carmen Piraino ${ }^{2}$, Roberto Mollo ${ }^{1}$, Stefano Calvieri ${ }^{3}$ \\ and Santi Maria Recupero ${ }^{4}$
}

\begin{abstract}
Background: Neurofibromatosis type 1 (NF1) is an autosomal dominant disorder involving aberrant proliferation of multiple tissues of neural crest origin. Retinal vascular alterations in NF1 have rarely been reported in the literature and their nature is not clear. This study describes distinctive retinal microvascular alterations and their relationship to choroidal nodules in patients with neurofibromatosis type 1.
\end{abstract}

Methods: This was a retrospective study where records of seventeen consecutive patients with diagnosis of NF1, presenting Lisch nodules and choroidal alterations, and 17 age and gender-matched healthy control patients were evaluated. Fundus photographs, near infrared reflectance and enhanced depth imaging - optical coherence tomography images were reviewed. Retinal microvascular abnormalities and choroidal and retinal alterations in proximity of the retinal microvacular alterations were carefully noted.

Results: 6 patients (35\%) presented distinctive microvascular abnormalities. These consisted of small, tortuous vessels with a "spiral" or "corckscrew" aspect. They were second or third order, small tributaries of the superior or inferior temporal vein. These vessels were all located overlying choroidal alterations as observed with near infrared reflectance. Enhanced depth imaging - optical coherence tomography showed alteration of choroidal vasculature due to the presence of choroidal nodules but otherwise retinal and choroidal cross-sections were unremarkable for morphology.

Conclusions: Retinal microvascular alterations overlying choroidal nodules in patients with NF1 can be considered another distinctive characteristic of the disease. Although the nature of these alterations is not clear, the authors speculate that functional disorders of vasomotor nerve cells, which originate in the embryonal neural crest can lead to their formation.

Keywords: Neurofibromatosis type 1, Retinal microvascular alterations, Near infrared reflectance, Optical coherence tomography-enhanced depth imaging, Phakomatoses

\section{Background}

Neurofibromatosis type 1 (NF1) is part of a group of heredofamilial disorders characterized by the presence of disseminated hamartoma for which Van der Hoeve introduced the termed "phakomatoses" in 1923 [1]. In ophthalmology hamartoma of the iris, termed Lisch nodules, are among the diagnostic criteria of NF1 [2]. However, choroidal hamartomas have also been proposed

\footnotetext{
* Correspondence: solmazzadeh@gmail.com

'Ophthalmology Unit, DAI Testa/Collo, Azienda Policlinico Umberto I,

University of Rome "Sapienza", viale del Policlinico 155, Rome, Italy
}

Full list of author information is available at the end of the article since they occur in 82 to $100 \%$ of cases [3,4]. In a unique report by Muci-Mendoza et al. retinal microvascular abnormalities were observed in $37.5 \%$ of patients [5]. The nature of these vessels is not clear, although it has been hypothesized that, due to their stable nature, they may be congenital. The present study was carried out to evaluate the presence of retinal microvascular abnormalities in patients with NF1 and to determine if these were associated with choroidal alterations.

\section{Methods}

All persons gave their informed consent prior to inclusion in the study. The research was given approval by 
the Institutional Review Board of the University of Rome "Sapienza" and was conducted in accordance with the tenets of the Declaration of Helsinki. Records of seventeen consecutive patients examined in 2014 with diagnosis of NF1 based on the stringent National Institutes of Health diagnostic criteria [6] were evaluated. Records of 17 age and gender-matched healthy control patients were also examined. Fundus photographs, near infrared reflectance (NIR) and enhanced depth imaging (EDI) optical coherence tomography (OCT) images acquired with the Spectralis OCT Family Acquisition Module, V 5.1.6.0 Heidelberg Engineering, Germany were reviewed. Retinal microvascular alterations and choroidal or retinal alterations in proximity of the microvascular alterations were carefully noted.

\section{Results}

Microvascular retinal abnormalities were identified in 6 patients (35\%) (3 men and 3 women, 42 to 57 years of age) in a total of 17 patients with NF1 presenting Lisch and choroidal nodules. No similar alterations were identified in the healthy control patients. The microvascular alterations were localized in the vascular arcade area and were second or third order, small tributaries of the superior or inferior temporal vein. They consisted of small tortuous vessels with a "spiral" or "corkscrew" appearance in 3 female and 2 male patients (Figure 1). One male patient, with extensive cutaneous neurofibromata, had 2 microvascular alterations of the superior and inferior temporal vein with a more complex "hemangioma-like" or "ball of thread" aspect (Figure 2). Fluorescein angiography images in this case showed that the microvascular alteration did not leak fluorescein. Visual acuity was 20/ 20 in all patients.

NIR images showed that the retinal microvessels were all overlying patchy choroidal alterations. On cross-sectional EDI-OCT images these alterations corresponded to hyperreflective choroidal nodules which occupied space in the choroidal thickness and gave an irregular profile to the choroidal vasculature.

\section{Discussion}

Retinal angiomatosis in a patient with NF1 was reported in 1967 by Frenkel [7]. Muci-Mendoza et al., in 2002, reported on 12 of 32 patients (37.5\%) who presented retinal microvascular abnormalities [5]. The present report is the second case series showing retinal microvascular alterations in six of 17 NF1 patients (35\%).

In the present study, all abnormal retinal microvessels were overlying patchy choroidal alterations as shown on NIR imaging. However, they were localized to the retina and did not involve the choroid, as shown by spectralis EDI-OCT cross-sections. Although choroidal vasculature is altered by the space occupying choroidal nodules, it is

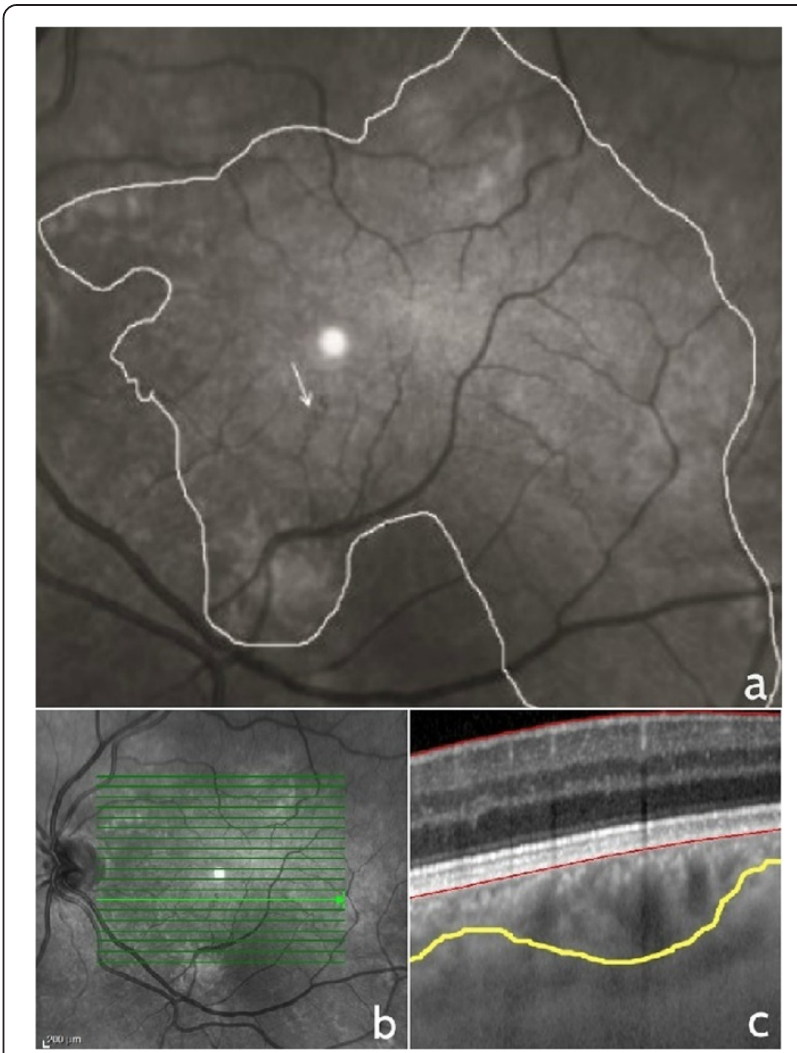

Figure 1 Near infrared reflectance and enhanced depth imaging optical coherence tomography cross-section of retinal and choroidal alterations in NF1. Near infrared reflectance image of "corkscrew" or "spiral" microvascular alteration (arrow) overlying large patchy choroidal alteration delineated with a white line (a), raster optical coherence tomography image and corresponding cross-section of the choroidal nodule delineated with a yellow line (b and c)

difficult to establish whether there is any correlation between these nodules and the abnormal microvascular retinal vessels.

Choroidal nodules are ovoidal bodies consisting of proliferating Schwann cells arranged in concentric rings around an axon [8]. They show similarities to cutaneous neurofibromata and Lisch nodules of the iris [9]. This follows the principle of aberrant proliferation of tissues of neural crest origin in NF1 [10] but does not directly justify the presence of vascular abnormalities, which can be an expression of changes in mesodermal germ layers. The term "phakomatoses", as described by Van der Hoeve, includes Recklinghausen's neurofibromatosis, tuberous sclerosis, Hippel-Lindau disease, Sturge-Weber syndrome, Louis-Bar syndrome and Wyburn-Mason syndrome [1]. Even before the modern era of DNA genomic studies, similarities in inheritance patterns and affected germ layers had been noted among the various phakomatoses. Today, there is still wide discussion regarding the interrelationships between these forms. Sporadic reports 


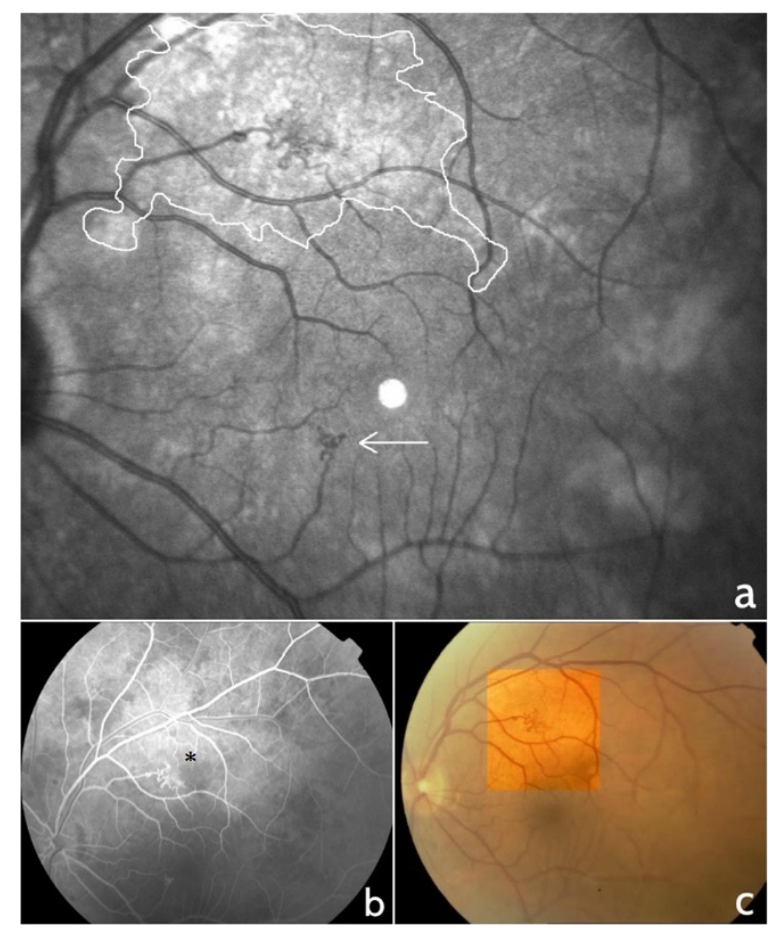

Figure 2 Retinal microvascular abnormality with "hemangioma-like" or "ball of thread" appearance in NF1. Near infrared reflectance image where the white line delineates the patchy choroidal alteration around the hemangioma-like microvascular abnormality and the arrow indicates a smaller microvascular abnormality (a), fluorescein angiography where the asterisk indicates the hemangioma-like microvascular abnormality (b) and fundus photograph with colour enhancement of the microvascular alteration (c).

in the literature show that a relationship can exist between a chiefly ectodermal and a primarily mesodermal phakomatosis and NF1 has been reported in association with von Hippel's disease [11,12]. Furthermore, in phakomatosis pigmentovascularis there is an association of vascular and pigmentary alterations $[13,14]$. There is evidence supporting developmental abnormalities of vasomotor nerve cells that originate in the embryonal neural crest and immunohistochemical studies have shown the presence of perivascular nerves in the port wine stains seen in Sturge-Weber Syndrome [15]. Although this is an extremely complex field where both embryogenesis and genomic analysis must be taken into consideration, it can be speculated that functional disorders of vasomotor nerve cells which originate in the embryonal neural crest can lead to the retinal microvascular alterations observed in NF1 patients.

\section{Conclusions}

This study describes "spiral" or "corkscrew" retinal microvascular alterations overlying choroidal nodules in patients with NF1. These alterations can be considered a distinctive retinal feature of NF1. Furthermore, as NF1 involves aberrant proliferation of multiple tissues of neural crest origin, these retinal alterations could possibly be related to functional disorders of vasomotor nerve cells.

\section{Abbreviations}

NF1: Neurofibromatosis type 1; NIR: Near infrared reflectance;

OCT: Optical coherence tomography; EDI: Enhanced depth imaging.

\section{Competing interests}

The authors declare that they have no competing interests.

\section{Authors' contributions}

SA made substantial contributions to the design and conception of the study, analysis and interpretation of OCT and NIR images, drafting and critical revision of the manuscript for important intellectual content. LF contributed to the conception of the study, acquisition of OCT and NIR images, interpretation of data and drafting of the manuscript. DCP contributed to the acquisition of fluorescein angiography images, preparation of figures and drafting of the manuscript. RM contributed to acquisition of OCT images and drafting of the manuscript. SC contributed to diagnosis of NF1, coordination of patients and drafting of the manuscript for dermatological aspects of NF1. SMR contributed to conception and design of the study and critical revision of the manuscript for important intellectual content. All authors read and approved the final version of the manuscript for publication.

\section{Acknowledgments}

The authors declare that they did not receive any funding for the present study.

\section{Author details}

'Ophthalmology Unit, DAI Testa/Collo, Azienda Policlinico Umberto I, University of Rome "Sapienza", viale del Policlinico 155, Rome, Italy. ${ }^{2}$ Ophthalmology Unit, Organi di Senso Department, University of Rome "Sapienza", viale del Policlinico 155, Rome, Italy. ${ }^{3}$ Dermatology Unit, University of Rome "Sapienza", viale del Policlinico 155, Rome, Italy. ${ }^{4}$ Ophthalmology Unit, Sant'Andrea Hospital, NESMOS Department, University of Rome "Sapienza", Via di Grottarossa 1035/1039, Rome, Italy.

Received: 19 July 2014 Accepted: 19 November 2014

Published: 25 November 2014

\section{References}

1. Van der Hoeve J: Eye diseases in tuberous sclerosis of the brain and in Recklinghausen's disease. Trans Ophthalmol Sci U K 1923, 43:534-541.

2. Recupero SM, Plateroti R, Abdolrahimzadeh S, De Dominicis M, Giustini S, Noviello MR, Calvieri S: Lisch nodules in neurofibromatosis type 1: relationship to age and cutaneous neurofibromas. Ann Ophthalmol 1996, 28:178-183.

3. Yasunari T, Shiraki K, Hattori H, Miki T: Frequency of choroidal abnormalities in neurofibromatosis type 1. Lancet 2000, 356:988-992.

4. Viola F, Villani E, Natacci F, Selicorni A, Melloni G, Vezzola D, Barteselli G, Mapelli C, Pirondini C, Ratiglia R: Choroidal abnormalities detected by near- infrared reflectance imaging as a new diagnostic criterion for neurofibromatosis 1. Ophthalmology 2012, 119:369-375.

5. Muci-Mendoza R, Ramella M, Fuenmayor R: Corkscrew retinal vessels in neurofibromatosis type 1: report of 12 cases. Br J Opthalmol 2002, 86:282-284

6. National Institutes of Health Consensus Development: Neurofibromatosis conference statement. Arch Neurol 1988, 45:575-578.

7. Frenkel M: Retinal angiomatosis in a patient with neurofibromatosis. Am J Ophthalmol 1967, 63:804-808.

8. Kurosawa A, Kurosawa H: Ovoid bodies in choroidal neurofibromatosis. Arch Ophthalmol 1982, 100:1939-1941.

9. Richetta A, Giustini S, Recupero SM, Pezza M, Carlomagno V, Amoruso G, Calvieri S: Lisch nodules of the iris in neurofibromatosis type 1. J Eur Acad Dermatol Venereol 2004, 18:342-344.

10. Kissel P, Andre JM, Jacquire A: The Neurocristopathies. New York: Masson; 1981. 
11. Thomas JV, Schwartz PL, Gragoudas ES: Von Hippel's disease in association with von Recklinghausen's neurofibromatosis. Br J Ophthalmol 1978, 62:604-608

12. Destro M, D'Amico D, Gragoudas ES, Brouekhurst RJ, Pinnolis MK, Albert DM, Topping TM, Puliafito CA: Retinal manifestations of neurofibromatosis. Arch Ophthalmol 1991, 109:662-666.

13. Recupero SM, Abdolrahimzadeh S, De Dominicis M, Mollo R: Sturge-Weber syndrome associated with naevus of Ota. Eye 1998, 12:212-213.

14. Noriega-Sanchez A, Makand ON, Herndon JH: Oculocutaneous melanosis associated with the Sturge-Weber syndrome. Neurology 1972, 22:256-262.

15. Smoller BR, Rosen S: Port-wine stains: a disease of altered neural modulation of blood vessels? Arch Dermatol 1986, 122:177-179.

doi:10.1186/1471-2415-14-146

Cite this article as: Abdolrahimzadeh et al:: Retinal microvascular

abnormalities overlying choroidal nodules in neurofibromatosis type 1 .

BMC Ophthalmology 2014 14:146.

\section{Submit your next manuscript to BioMed Central and take full advantage of:}

- Convenient online submission

- Thorough peer review

- No space constraints or color figure charges

- Immediate publication on acceptance

- Inclusion in PubMed, CAS, Scopus and Google Scholar

- Research which is freely available for redistribution 